\title{
Thrombus histopathology from thrombectomy, an important secondary prevention tool?
}

V. Pignato1, L. Lebrun2, B. Lubicz3, B. Mine3, J. Vazquez Suarez3, N. Ligot1, G. Naeije1.

1ULB-Hôpital Erasme, Neurology, BRUXELLES, Belgium.

2ULB-Hôpital Erasme, Anatomo-pathology, Bruxelles, Belgium.

3ULB-Hôpital Erasme, Neurointerventional radiology, Bruxelles,

Belgium.

Abstract reference number: AS03-020

\section{Background and aims}

Atrial fibrillation (AF) is responsible for substantial proportion ischemic strokes (IS) and need in some cases a prolonged cardiac heart rate monitoring to be detected, sometimes months after stroke index. However, optimal secondary prevention for cardioembolic (CE) stroke requires early initiation of anticoagulant therapy.

We aimed in this study to assess if thrombus histopathology (TH) was reliable enough to base early specific stroke secondary prevention therapy upon its results.

\section{Method}

35 patients who had underwent thrombectomy for IS due to proximal occlusion and TH examination were included. Stroke etiologies based on TOAST classification at hospital discharge after ECG, 24h-telemetry and cardiac echography, and at follow-up after ambulatory $24 \mathrm{~h}-\mathrm{ECG}$ were compared to TH diagnosis. Positive and negative predictive values of TH for CE strokes were calculated.

\section{Results}

Of the 35 thrombi analyzed, 24 had a CE histology. Among these 24 patients, 13 early-proved AF based on the initial ECG/24h-telemetry were found and the follow-up 24h-ECG allowed to record AF in 6 more patients. $11 \mathrm{TH}$ were classified as unknown or atherothrombotic, of those follow-up disclosed CE stroke in 6.

TH has positive and negative predictive values of $87,5 \%$ and $45 \%$ for CE strokes, respectively.

\section{Conclusion}

TH may be useful to identify earlier patients with a CE stroke, help giving a more accurate secondary prevention treatment in a substantial proportion of patients and prevent foreseeable recurrences. Further studies including more patients are needed to strongly demonstrate the $\mathrm{TH}$ reliability to base the stroke secondary prevention treatment early decision.

\begin{tabular}{|c|c|c|c|}
\hline & CE stroke & NCE stroke & \\
\hline CE Histology & 21 & 3 & 24 \\
\hline NCE Histology & 6 & 5 & 11 \\
\hline & 27 & 8 & TOTAL $=35$ \\
\hline \multicolumn{4}{|c|}{$\begin{array}{l}{ }^{*} \mathrm{CE}=\text { cardioembolic } \\
{ }^{*} \mathrm{NCE}=\text { noncardioembolic }\end{array}$} \\
\hline \multicolumn{2}{|c|}{$P P V=87,5 \%$} & \multicolumn{2}{|c|}{$N P V=45 \%$} \\
\hline
\end{tabular}
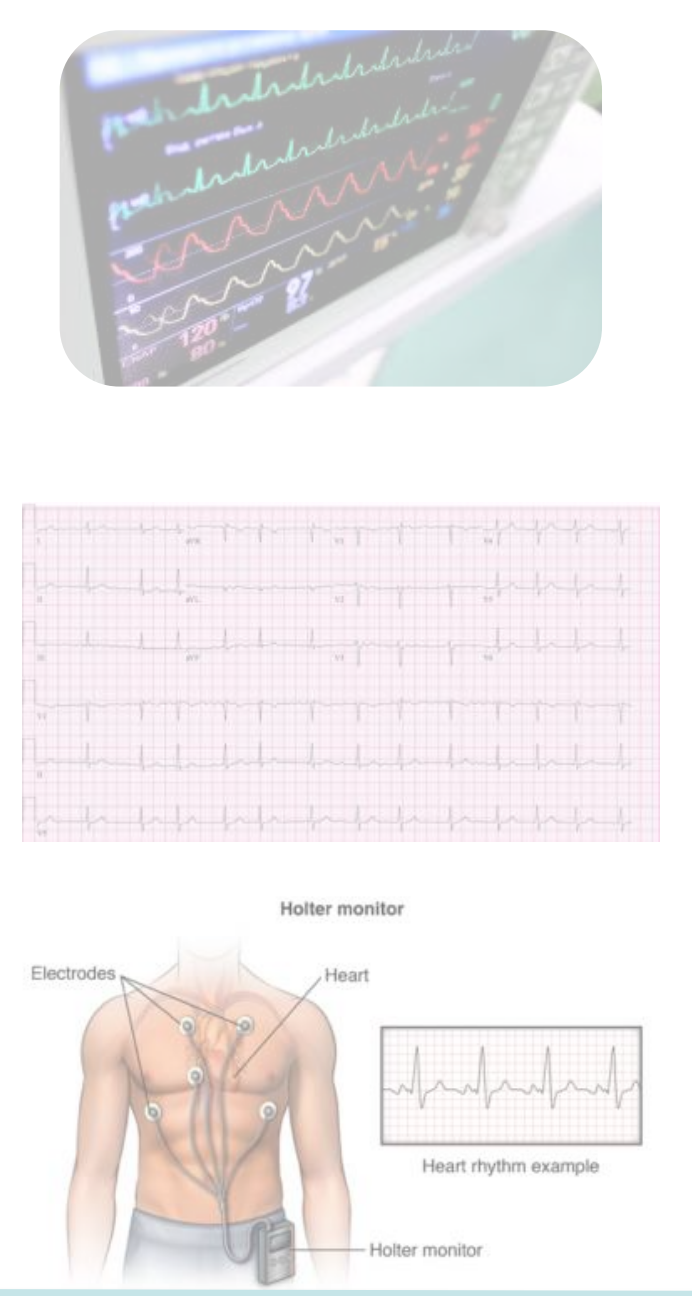

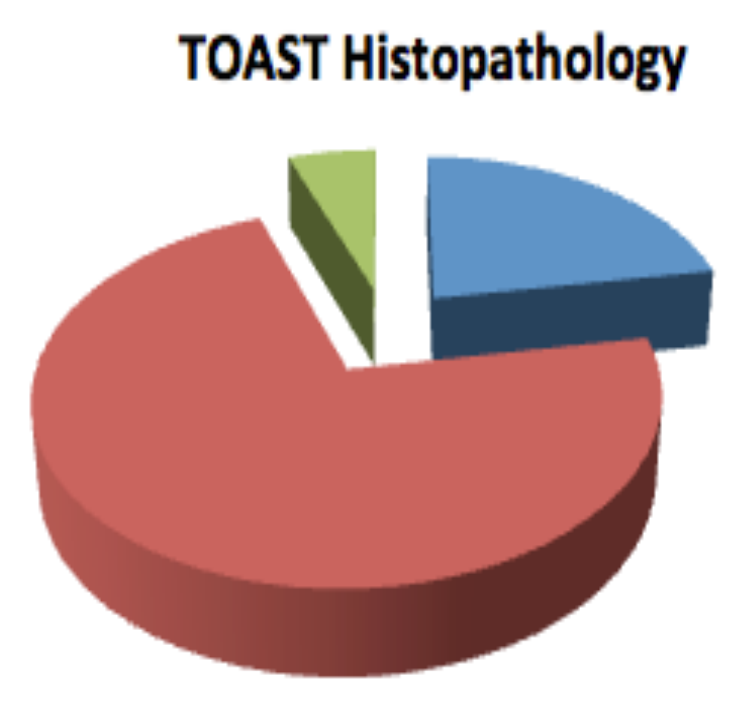

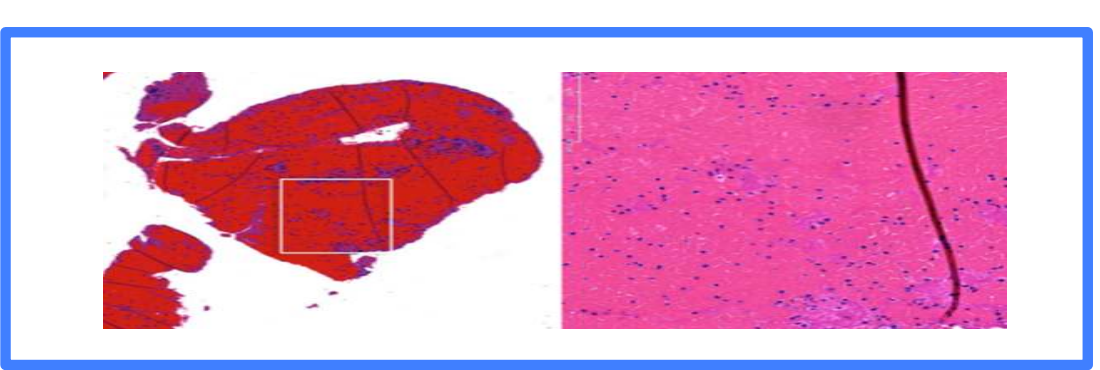

Atherothrombotic histology

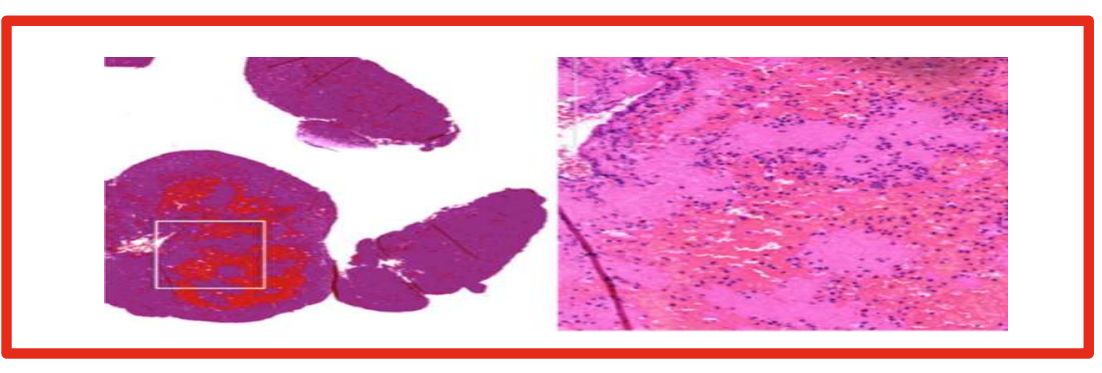

Cardioembolic histology

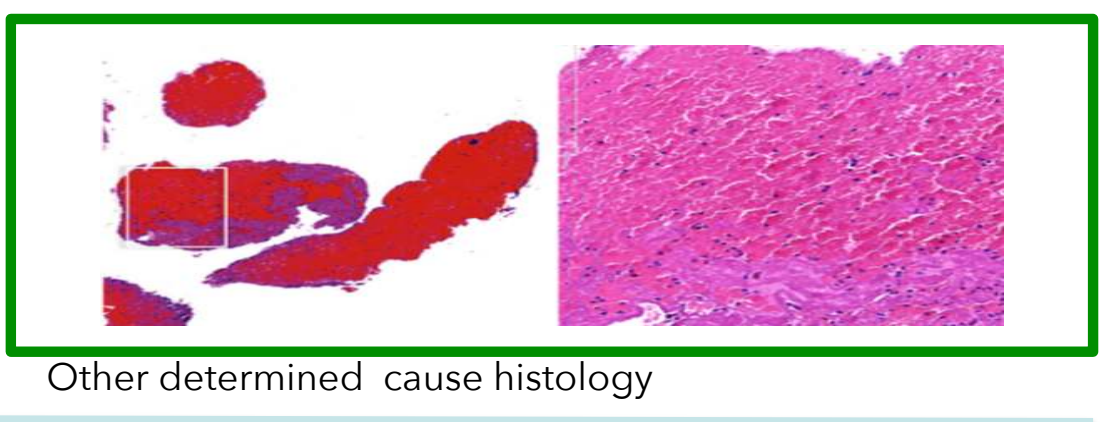

